\title{
Toward Mixed-Media Design Studios
}

\section{Florian Geyer}

University of Konstanz | florian.geyer@uni-konstanz.de

\section{Harald Reiterer}

University of Konstanz | harald.reiterer@uni-konstanz.de

"We are far from seeing a fully digital design studio. Unfortunately, the modern hybrid studio_-straddling both digital and traditional methods-can experience new challenges along with the new tools" [1].

In many design studios, dedicated rooms with abundant display space are used for colocated design collaboration. And this is for good reasons. Externalizing and sharing design knowledge are key activities of everyday design practice. We share our design ideas or inspirational artifacts, such as paper sketches, printouts, and whiteboard drawings, in a spatial and informal way to invite comments and feedback within the design team. The physical display space in design studios thereby serves as a powerful tool that extends our social and cognitive abilities, including remembering, self-assessment, associating, and judging. Material artifacts assist as mediators for implicit communication between designers, developers, and other stakeholders involved in the design process. The spatiality of these physical representations and having things observable and in reach also facilitate a visual and embodied form of thinking. Being able to group and regroup artifacts in a direct and physical way allows us to identify relations, tensions, or analogies between the collected pieces [2].

Keeping artifacts in a collaborative space can also trigger free associations between knowledge from different design projects, which may lead to new and unexpected insights or ideas. In a way, these spatial arrangements are a tool for thinking and reflection-making cooperative design knowledge and the progress of the design process visible.

Despite the manifold technological advancements we have witnessed in recent years, we still experience bottlenecks when digital design artifacts are used in the design process. Their representations are bound to digital tools that typically do not facilitate or invite the kinds of interactions described here. While digital objects such as images, videos, and websites are capable of representing dynamics and are easy to share and archive, they do not have the unique affordances of material artifacts. Due to the predominant desktop computing paradigm, digital representations often remain hidden in file systems. Furthermore, many digital tools are designed for individual use, isolating designers behind screens, leading to a breakdown of communication that is vital for a shared understanding in the group. Rigid interaction models or formal interaction techniques and modalities may further impose limitations not only on the spatial characteristics of design practice, but also on other important aspects such as embodiment of thought, the workflow of design methods, as well as coordination and communication.

Due to this dualism of digital and material design artifacts and their respective qualities for designers, transitions between both worlds are quite common. These transitions, however, may harm the overall flow of the design process. As a result, designers often consider technology to be harmful in their collaborative work environments and stick to traditional media. By using physical artifacts and by harnessing the spatial properties of the design studio environment, they can make use of rich forms of expression like body language, facial expressions, and the immediacy of verbal communication that are crucial for expressing their creativity. However, at the same time they give up on potential benefits of using digital media, such as ease of documenta- 
tion, sharing, and reuse. Both the strengths and limitations of physical design practice point to the need for an integration of computational functionality with the physical and spatial ecology for better supporting collaborative design work. As long as digital design tools do not consider and actively support these aspects, they will hardly be adopted for use in practice.

\section{Interactive Spaces for}

\section{Collaborative Design Practices}

In HCI we have a growing interest in technologies that integrate with elements of the physical environment such as space and material artifacts. Tangible interfaces and the notion of "reality-based interaction" [3] are also promising paths for supporting collaborative design practices. In that sense, the power of digital tools and informal interaction techniques could be used to augment collaborative design activities instead of replacing them with digital-only tools. As we outline here, this can be achieved by carefully designing digital functionality with the goal of respecting and preserving wellestablished characteristics of traditional practice. In our research we have mainly explored three themes toward a better integration of digital media in collaborative design practice: hybrid artifacts, interactive display space, and capture and visualization.

\section{Externalizing with hybrid design} artifacts. The material nature of design artifacts is one of the most obvious benefits of physical practice. The rich affordances of paper sketches, Post-its, and printouts support designers in rapid externalization and invite group interactions. When interacting with artifacts in design sessions, the properties of material representations allow us to view, to gesture on, to navigate between, and to annotate them in a fluent way. Achieving similar qualities with digital tools requires a simulation or imitation of these characteristics. Recently, we have seen great progress toward mixedmedia design tools that make use of both physical and digital representations, thereby reducing the burden of transforming artifacts between these two worlds.

In our research we have developed AffinityTable [4], a hybrid surface for supporting the affinity diagramming method (see Figure 2). This interactive workspace, based on a large multitouch table and a
- Figure 1. Affinity diagramming and sketching methods are classic examples of the material and spatial nature of collaborative design that is practiced in design studios.

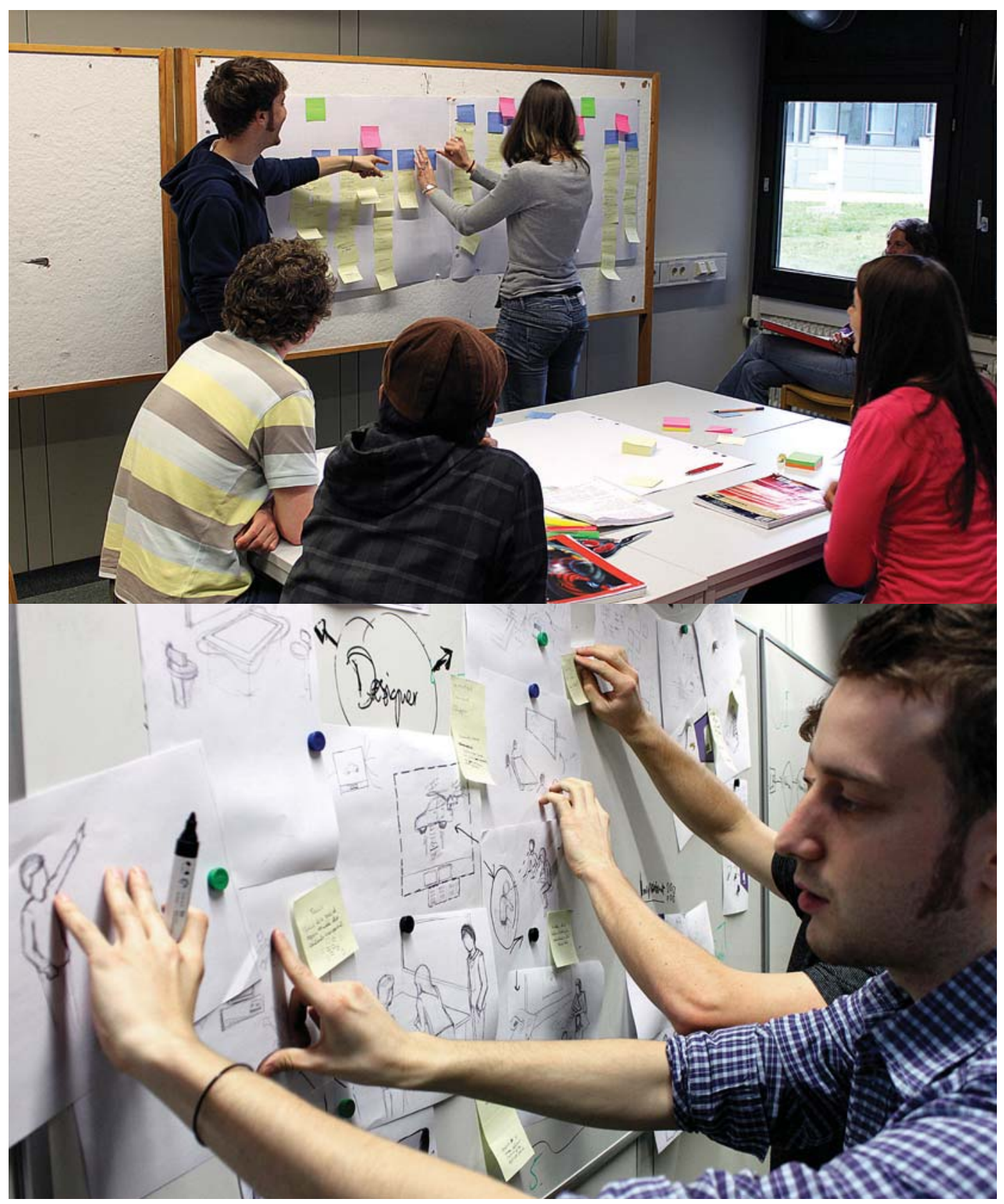




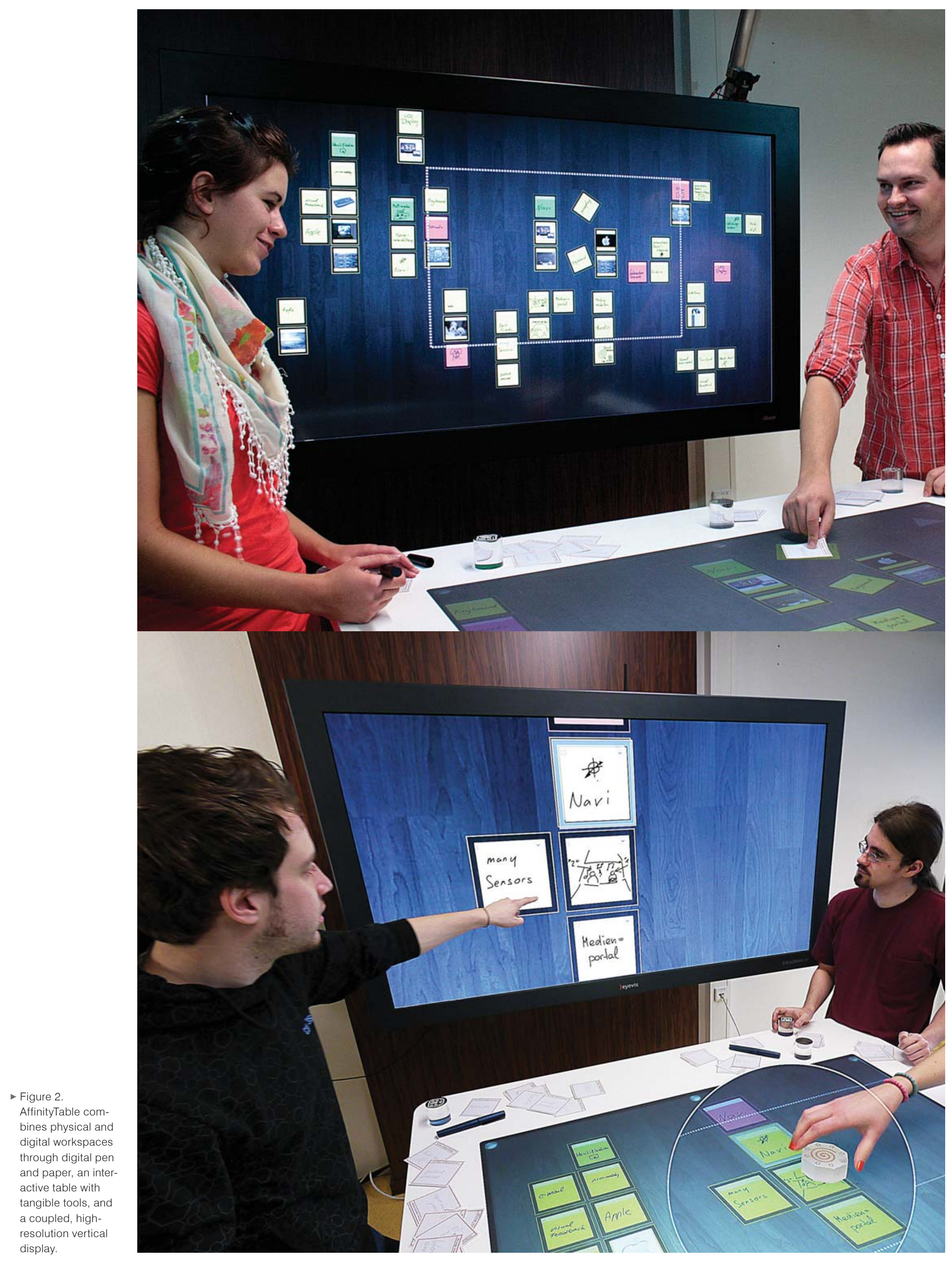




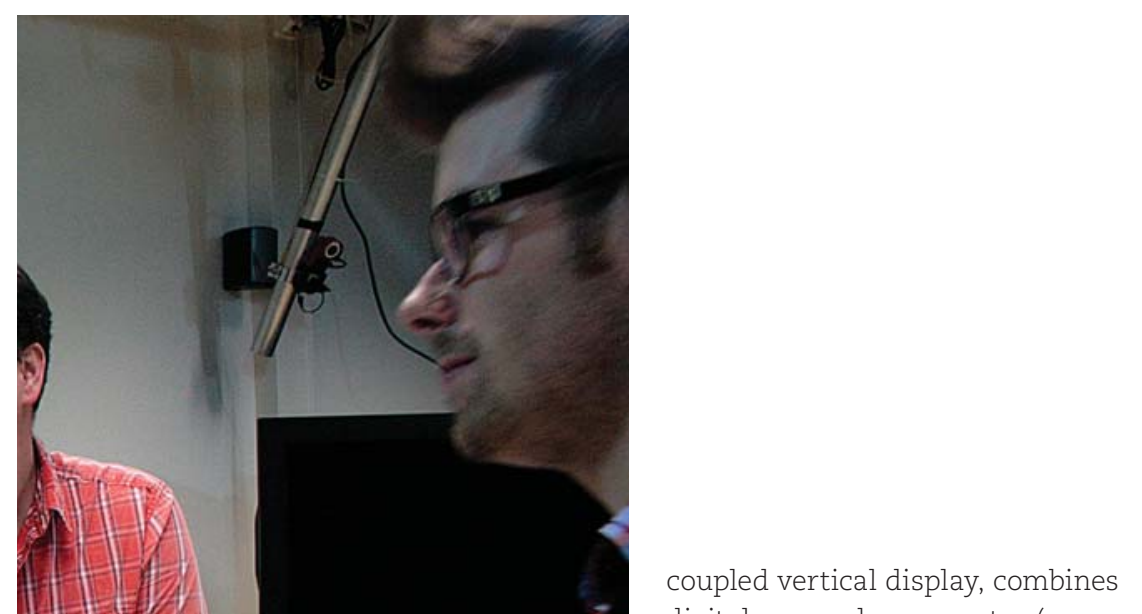

digital pen and paper notes (see www.anoto.com), tangible tools, and multitouch interaction techniques to replicate many of the crucial qualities of material artifacts. At the same time, it extends the traditional methods by providing functionalities that complement the original workflow, such as image retrieval based on handwritten text, search functions, highlighting of artifacts for discussion, and more efficient clustering mechanisms. In our user studies we could confirm the benefits of physical material, in particular for externalization activities, while we found that digital functionality was especially useful in convergent activities like sensemaking and discussion.

Collaborative thinking with interactive display space. As outlined here, display space and spatial arrangements of artifacts are important tools for thinking and communication within a design team. By leveraging the ability to physically align, juxtapose, and compare different artifacts in space, designers harness their spatial thinking abilities. Consequently, it may also be valuable to explore the role of digital display space for use in collaborative design activities. As multi-display environments and large interactive displays such as interactive whiteboards, multitouch tables, and wallsize screens become increasingly available, this ecology of screens can be used to augment the spatial nature of design practice. In the past we have seen significant advances in integrating displays into a larger infrastructure, such as rooms or furniture, to support more creative work styles and collaborative sensemaking. In these systems, interactive displays are used for presenting, discussing, and annotating artifacts during meetings [1].
In our research we also explored the role that multiple displays can play in navigation and spatial positioning of digital design artifacts [5]. In our AffinityTable system, we coupled two large displays within a virtual workspace, which enables us to provide multiple views on collections of digital artifacts. For example, we display a detailed view of notes on the table, while the vertical display presents an overview of all available notes, thereby facilitating an overview on the progress of the design activity (see Figure 2, top). As a result of this division, we also implicitly assigned roles to these workspaces, with the table primarily used for manipulations (action space), while the vertical display supports reflection activities (reflection space). Furthermore, we designed tangible tools that can be used for generating dynamic views of groups of artifacts. By selecting a region on the table with a token object, this area within the virtual workspace is then enlarged on the wall display (see Figure 2, bottom). This zooming functionality can further be refined by turning the token to increase or decrease the degree of magnification. Designers may use this functionality to focus on specific artifacts during discussion. From our experiences, the dynamic nature of interactive displays can be used to augment our spatial-thinking abilities and aid group coordination and awareness. This allows us not only to imitate the spatial characteristics of traditional practice but also to go beyond what is possible with material artifacts.

Reflecting by capturing and visualizing the design process. Our physical design practice has a lot of benefits but also some drawbacks. One of the most crucial drawbacks is the tremendous effort required for documenting, archiving, and sharing 


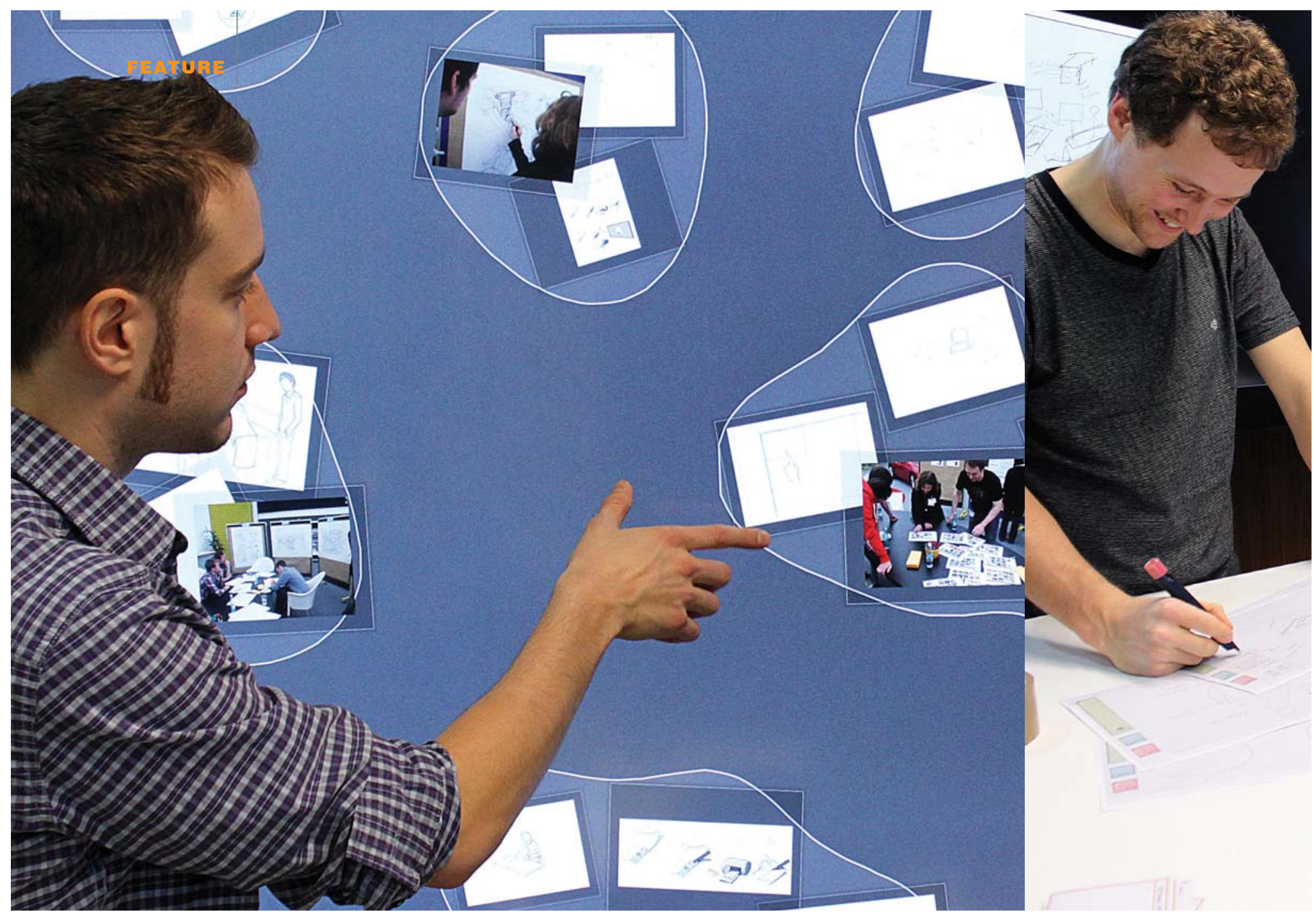

- Figure 3. We designed an organic interactive visualization called Blub (left) for supporting spatial reflection and sense-making tasks and the hyperbolic tree SketchVis (right) for capturing, visualizing, and interacting with design session histories. design artifacts over a longer period of time. Physical display space eventually needs to be cleared if the number of artifacts grows too large. Setting up new displays and clearing old ones impedes the reuse of design knowledge in later stages of a design process or across different design projects. Due to the dynamic nature of creative group work, organizing and archiving individual contributions in a formal structure is an awkward task. Technology gives us the ability to capture, store, and retrieve a virtually unlimited amount of design content in digital repositories or databases. It also allows us to capture and replay the history of design activities and compare and reflect over multiple steps and decisions of a complete design process. Just recently, dedicated tools emerged that are particularly designed for these activities, supporting situated reflection associated with digital or physical artifacts.

In our research we explore the role of spatial visualizations in the context of reflection activities. For example, we adapted interactive bubble visualizations [6] for Blub, a zoomable interface for grouping design artifacts on interactive surfaces such as multitouch tables or walls (see Figure 3, left). Dynamic bubbles thereby serve the purpose of creating collections of digital artifacts in organic groups. Our goal with this visualization is to augment reflection activities for better identifying relations, tensions, or analogies between the grouped artifacts. In a different context, we designed the visualization SketchVis, which can be used to capture and visualize histories of collaborative design sessions. The tree-like visualization is dynamically generated by analyzing live sketching activities performed with digital pen and paper (see Figure 3, right). Data retrieved from the digital pens such as strokes, pages, and pen identification are used for displaying an interactive hyperbolic tree, conveying information about the number of unique ideas, relations and links between ideas, annotations, and individual designers, as well as awareness of currently manipulated artifacts. In our user studies with creative practitioners, we found these kinds of visualizations are especially useful for creative facilitators during or after the sessions to analyze and improve the productivity of such activities.

\section{Designing Mixed-Media Work \\ Environments}

The concepts we have discussed here represent small steps toward a larger vision of mixed-media design studios and the design of interactive spaces in general. We imagine that future work environments might integrate a multitude of the presented techniques with the ecology of tools and activities of real-life work practice in a complementary way-not only for design activities but for collaborative sense-making and thinking in general. Many 
